\title{
Estimating cost savings of pharmacogenetic testing for depression in real-world clinical settings
}

This article was published in the following Dove Press journal:

Neuropsychiatric Disease and Treatment

\author{
Alejandra Maciel \\ Ali Cullors \\ Andrew A Lukowiak \\ Jorge Garces \\ AltheaDx, San Diego, CA, USA
}

Correspondence: Alejandra Maciel AltheaDx, 10578 Science Center Drive, San Diego, CA 92121, USA

Email amaciel@altheadx.com

\begin{abstract}
The burden of depression significantly impacts the patient, the health care system, and society, at large. Medication management guided by pharmacogenetics has been shown to increase therapeutic efficacy and improve symptoms in patients diagnosed with depression, but limited data are available on the cost savings of pharmacogenetic-guided interventions outside of psychiatric clinical specialties. Our study utilizes published health care costs and clinical patient outcome data to model the economic impact of pharmacogenetic-guided treatment for depression in a variety of clinical settings. Assuming a test cost of USD $\$ 2,000$ for pharmacogenetic testing, the model predicts a savings of USD \$3,962 annually per patient with pharmacogenetic-guided medication management.
\end{abstract}

Keywords: pharmacogenetics, depression, personalized medicine, cost savings, pharmacoeconomics, psychotropic, cytochrome $\mathrm{P} 450$

\section{Introduction}

Depression is a chronic psychiatric disorder with high prevalence and low treatment response rates, which significantly burdens the patient, the medical provider, and society. It affects 16 million adults ages 15-44 years of age in the $\mathrm{USA}^{1}$ and is associated with an increased risk of cardiovascular disease, all-cause mortality, ${ }^{2}$ and reduced quality of life. ${ }^{3}$ Studies show that depression is an independent risk factor for coronary artery disease and sudden cardiac death, ${ }^{4-6}$ leading to a 7-11 years reduction in life expectancy, similar to lifetime smokers. ${ }^{6}$ The burden of depression is far reaching and extends beyond the individual. Epidemiological studies point to a consistent association between depression and productivity loss. Individuals with depression are 5 times more likely to miss work due to illness ${ }^{7}$ and 4.78 times more likely to be on disability. ${ }^{8}$ These numbers place depression as the leading cause of disability in the USA, amassing an estimated 400 million days of disability per year. ${ }^{9}$ Depression is also associated with a $50 \%-75 \%$ increase in health care utilization, ${ }^{10}$ a cost that surpassed USD $\$ 98$ billion in 2010. ${ }^{9}$ When the costs of indirect services and lost productivity are included, the economic burden of individuals with depression totals USD $\$ 210.5$ billion per year. ${ }^{9}$

The availability of new medications has contributed to a $400 \%$ increase in antidepressant use in the last 20 years, ranking antidepressants the third most commonly prescribed drug type among all adults. ${ }^{11}$ With over 35 FDA-approved antidepressants in the market ${ }^{12}$ and multiple dosing possibilities, health care providers currently rely on trial and error methods to select and manage medications. ${ }^{13,14}$ This approach results in over two-thirds of users failing first treatment. ${ }^{15}$ For these users, each additional treatment failure lowers the probability of remission and increases the probability of relapse. ${ }^{15,16}$ In addition, nonresponders are disproportionately burdened by adverse 
effects compared to patients who achieve remission, with almost $90 \%$ of those experiencing serious adverse effects unable to achieve remission. ${ }^{15}$ The rising prevalence of depression, new screening recommendations by the US Task Force, and a nationwide shortage of psychiatrists are factors contributing to a shift in the management of mental health. Currently, $96 \%$ of US counties have an unmet need for mental health professionals, equivalent to a deficit of 4,000 psychiatrists. ${ }^{17}$ As nearly $60 \%$ of psychiatrists enter retirement age, the shortage of psychiatrists is only expected to widen. ${ }^{18}$ With a lifetime prevalence of major depressive disorder (MDD) of $16.6 \%$ and a critical shortage of psychiatrists, a wide spectrum of health care providers are now treating neuropsychiatric disorders. ${ }^{19,20}$ In fact, $70 \%-80 \%$ of prescriptions for psychiatric medication are written by family medicine practitioners, primary care physicians, internal medicine practitioners, and obstetrician/ gynecologists. $^{20}$

Adopting new technologies that improve patient outcomes in a variety of clinical settings could ease the high cost and challenges of managing depression. NeuroIDgenetix $^{\circledR}$ testing is a promising tool in personalized medicine that has been shown to optimize medication management for depression or anxiety patients by using genetic factors and current drug regimen to determine variations in clinical response and side effects. ${ }^{21,22}$ The NeuroIDgenetix test uses a genetic variant panel of 10 genes, along with concomitant medications, to make medication management recommendations based on gene-drug and drug-drug interactions for over 40 medications used in the treatment of depression and anxiety. This technology can help mental health providers predict medication response and appropriate dosing, leading to improved patient outcomes. In the largest randomized, double-blind clinical trial conducted to date, Bradley et al enrolled 685 patients diagnosed with depression and/or anxiety in a variety of clinical settings that included psychiatric and nonpsychiatric clinics. The researchers found significantly higher remission rates for depression patients with medication management guided by NeuroIDgenetix compared with patients with medication management per standard of care $(35 \%$ vs $13 \%, P=0.02) .{ }^{21}$ The NeuroIDgenetix-guided group also achieved significantly higher response rates compared with standard of care (73\% vs 36\%, $P=0.001) .{ }^{21}$

Our analysis uses clinical patient outcome data from Bradley et al and published health care costs to model the cost savings associated with pharmacogenetic-guided treatment for depression in Psychiatry, Internal Medicine, Obstetrics \& Gynecology, and Family Medicine clinics.

\section{Methods}

The treatment response rates obtained from the clinical study reported by Bradley et al were inputted into an MDD cost analysis model created by Greenberg et al to assess the total economic burden of MDD. Our analysis explores the cost savings potentially associated with pharmacogenetic-guided management of patients clinically diagnosed with depression based on treatment response.

\section{Ethics approval}

All analyses were conducted with data obtained from published, peer-reviewed articles. The cost data reported by Greenberg et al used national survey and administrative claims data. The clinical efficacy data reported by Bradley et al were results from the randomized clinical trial with Institutional Review Board (IRB) approval and patient consent. As this study did not involve the collection, use, or transmittal of individually identifiable data, IRB review or approval was not required.

\section{Treatment response rates}

The treatment response rates are findings reported by Bradley et al in the double-blind, multicenter, randomized clinical trial that determined that pharmacogenetics-guided treatment improved clinical outcomes for patients with depression or anxiety disorders (ClinicalTrials.gov Identifier NCT02878928). ${ }^{21}$ The trial enrolled 685 consented adults from 20 independent clinical sites that included Psychiatry, Internal Medicine, Obstetrics \& Gynecology, and Family Medicine. Patients with a diagnosis of depression or anxiety were included in the study if they were new to treatment, had failing treatment, or had experienced adverse events due to psychiatric medications. Patients were randomized by disease and severity to the experimental group (medication management guided by the NeuroIDgenetix test) or the control group (medication management per standard of care). Buccal cells were collected from all patients during screening. For those within the experimental group, the NeuroIDgenetix test reports were released to participating clinicians to help guide medication decisions. For the control group, the NeuroIDgenetix results were withheld and clinicians were asked to manage control patients according to the standard of care. Patients were monitored for depression or anxiety symptoms using the appropriate Hamilton Rating Scale (HRS) at 4, 8, and 12 weeks by an independent reviewer (rater). Disorder severity was classified based on total HRS score: 0-7, normal; 8-17, mild disorder; 18-24, moderate disorder; and $>24$, severe disorder. Subjects and raters were blinded to study arm assignment. 
Table I Depression clinical outcomes at week 12

\begin{tabular}{lll}
\hline & Remitters, \% & Nonresponders, \% \\
\hline $\begin{array}{l}\text { NeurolDgenetix }{ }^{\circledR} \text { guided } \\
\text { (experimental control) }\end{array}$ & 35 & 27 \\
Standard of care (control group) & 13 & 64 \\
\hline Note: Data from Bradey et l. $^{21}$ & &
\end{tabular}

Note: Data from Bradley et al. ${ }^{21}$

Among subjects with severe depression, the study found significantly higher remission rates for the experimental group compared to control ( $35 \%$ vs $13 \%, P=0.02$ ) after 12 weeks (Table 1). Similarly, experimental subjects achieved $73 \%$ response rates, compared to $36 \%$ in the control group $(P=0.001){ }^{21}$

\section{Cost data}

We used the total per-patient economic burden of MDD published by Greenberg et al in 2015 , as it provides a comprehensive overall cost of depression and details the breakdown of factors included in each cost category. The total economic burden of an individual with MDD according to Greenberg is the sum of the direct health care costs associated with MDD (such as costs of medical services and prescriptions), the costs associated with depression other than MDD (costs of non-depression mental health), the costs of non-mental health medical services and prescription, and the costs of missed productivity (absenteeism, presenteeism, and disability; Figure 1). ${ }^{9}$ Data were collected from a private insurance database with 16 million beneficiaries from 69 large, self-insured US companies. Patients with a minimum of two ICD-9 claims for MDD and with continuous health care eligibility were included in the analysis. Patients in the control group were selected using similar criteria but were not on antidepressants or had any MDD-related claims. ${ }^{9}$ The costs reported by Greenberg et al reflect all costs paid for service including patient out-of-pocket costs. ${ }^{9}$

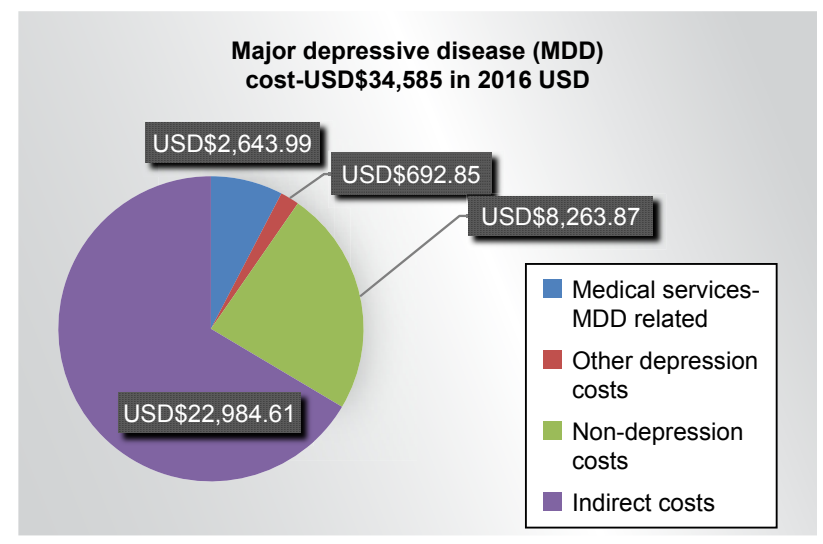

Figure I Major depressive disorder cost.

Note: Data retrieved from Greenberg et $\mathrm{al}^{9}$ to show which costs were included and converted to 2016 USD\$.

\section{Prescription data}

Greenberg et al identified three sources of prescription costs for patients with depression: MDD-related, other depression-related, and non-depression-related costs. Our model applied only the MDD-related prescription costs to nonresponders in order to maintain a more conservative model of cost savings. The USD $\$ 955$ MDD-related prescription cost was converted to 2016 dollars using consumer price index (CPI) data available in table $3 \mathrm{~A}$ of the CPI-Medical Care Component.

\section{Results}

The annual, per-patient economic burden of MDD reported by Greenberg was US $\$ 30,949$ compared to US $\$ 5,744$ for the control group in the year 2012. ${ }^{9}$ For our model, we assigned Greenberg's cost of MDD patients to the nonresponder group in the Bradley study. Similarly, Greenberg's cost for the control group was assigned to remitters in the Bradley study, but adjusted to include MDD-related pharmacy costs. After adjusting for inflation to 2016 dollars, the costs totaled US $\$ 34,585$ per nonresponder and US $\$ 6,419$ per remitter. The individuals in the control group used by Greenberg matched the MDD group in Bradley but were not on MDD-related medications. ${ }^{9}$ Because remitters differ from healthy (control) patients in the fact that the former are on MDD-related medications, we added the same MDD prescription costs associated with nonresponders (US $\$ 1,067$ in 2016) to remitters, which yielded a total per-patient cost of US\$7,486 for remitters (Table 2).

The percent difference in remission rates between the control and experimental groups was calculated at $22 \%$ as reported by Bradley et al (Table 3). ${ }^{9}$ This value was multiplied by the difference in cost between a remission patient and a nonrespondent patient (USD\$27,099) to obtain the cost savings associated with the use of the NeuroIDgenetix test in the treatment of depression (Figure 2). The model resulted in a savings of USD $\$ 5,962$ annually per patient tested. Including the price of testing at USD $\$ 2,000$, the cost savings for pharmacogenetic-testing vs standard of care totaled USD\$3,962 per year.

\section{Discussion}

Previous studies have presented data pointing to increased efficacy by incorporating pharmacogenetic testing in the

Table 2 Annualized cost of MDD management in 2016 by treatment status

\begin{tabular}{llll}
\hline & Remitter & Nonresponder & Difference in cost \\
\hline Estimated cost & USD $\$ 7,486$ & USD $\$ 34,585$ & USD $\$ 27,099$ \\
\hline
\end{tabular}

Abbreviation: MDD, major depressive disorder. 
Table 3 Patients requiring treatment

\begin{tabular}{|c|c|c|c|}
\hline & Neurol & Control, \% & Difference, \% \\
\hline & Dgenetix, \% & & \\
\hline $\begin{array}{l}\text { Patients requiring } \\
\text { treatment (non-remitters) }\end{array}$ & 65 & 87 & 22 \\
\hline
\end{tabular}

Note: Data from Bradley et al. ${ }^{2 !}$

management of patients diagnosed with depression. ${ }^{21,23}$ Other studies also point to decreased adverse drug events, ${ }^{22,25}$ lower health care utilization costs, ${ }^{24,26,27}$ and increased adherence rates with pharmacogenetic-guided treatment. ${ }^{27}$ Our model shows a potential annual cost savings of USD $\$ 3,962$ per patient tested with NeuroIDgenetix. The multicenter, randomized clinical study referenced in this analysis was designed to measure differences in efficacy of treatment and not powered to observe economic differences between cohorts. We, therefore, have used published data in conjunction with our clinical findings to model the potential cost savings of incorporating pharmacogenetic-guided treatment to psychiatric care in a variety of clinical settings. While the cost savings presented in this model are similar to those reported in previous studies (Table 4), we believe the broad-based modeling used to calculate our cost savings is a conservative estimate. For example, we found that in an analysis of all patients enrolled in the study, incorporating the NeuroIDgenetix test in the medication management of depression or anxiety patients resulted in a net reduction of 1.21 disability days per patient per month. Using the disability claims costs reported by Greenberg and adjusting to 2015 wages based on the National Average Wage Indexing Series, we calculated a monthly disability-related cost savings

\section{Depression patients requiring treatment}

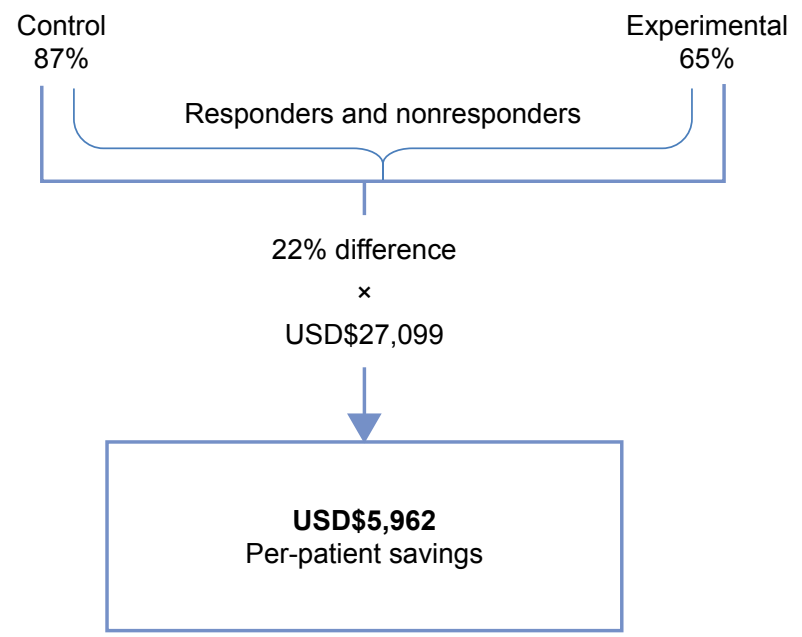

Figure 2 Cost-savings calculation.
Table 4 Published per-patient savings using genetic testing to guide medication management (adjusted to 2016 dollars)

\begin{tabular}{llll}
\hline & $\begin{array}{l}\text { Present } \\
\text { findings 2016 }\end{array}$ & $\begin{array}{l}\text { Winner } \\
\text { et al }{ }^{27}\end{array}$ & ${\text { Chou et a }\left.\right|^{28}}^{2}$ \\
\hline Per-patient savings & USD $\$ 5,962$ & USD\$6,193 & $\begin{array}{l}\text { USD } \$ 7,112- \\
\text { USD } \$ 10,667\end{array}$ \\
\hline
\end{tabular}

of USD $\$ 121.13$ or USD $\$ 1,453.56$ annually per patient with NeuroIDgenetix-guided medication management, compared to Greenberg's disability-related costs of USD $\$ 967$ per patient (adjusted for 2016). This points to potential greater savings than those reported in our model. Additionally, our model does not account for patients who responded to antidepressant medications, but did not remit during the clinical study. These patients most likely incur additional savings; however, the savings are not quantified in Greenberg's original analysis and are therefore not included in this model.

Lastly, this model differs from previous cost-savings models in various ways. Winner et al conducted a retrospective chart review and found that pharmacogenetic testing could identify suboptimal medication regimens that could lead to cost savings of USD $\$ 6,193$ (adjusted for 2016 dollars) for depression and anxiety patients. ${ }^{27}$ The analysis compared the costs associated with "red bin" patients (those with medications identified by their report as most problematic) to "green bin" (use as directed) or "yellow bin" (use with caution) patients. Given the retrospective nature of their approach, it is difficult to discern whether the higher costs associated with "red bin" patients are due to suboptimal medication regimens or intrinsic group characteristics. For example, it is possible that patients in the red bin category suffered from more severe depression than those in the green or yellow category. For this reason, we chose to use data from a randomized clinical trial and apply economic data from Greenberg. In a different study, Chou et al calculated cost savings based on total hospitalization days and total costs observed over 1 year for patients admitted to a psychiatric hospital. Because Chou et al restricted their analysis to patients with extreme CYP2D6 activity (ultrarapid metabolizers and poor metabolizers) and psychiatric inpatients, a population with more severe depression and higher baseline costs than our study, we feel that our model better approximates cost savings associated with pharmacogenetic testing in real-world medical practice. ${ }^{28}$

This study is a first step in modeling the potential cost savings associated with the implementation of pharmacogenetics in patients with depression and/or anxiety from a societal perspective. This study has potential limitations that are important to note and should be addressed in future studies. First, 
this model utilizes data published in peer-reviewed journals to calculate annual cost savings associated with pharmacogenetic testing. As such, clinical efficacy and cost data are derived from two different sources. While not ideal, the cost savings modeled in this study are in line with previous studies, providing support to our findings that pharmacogeneticguided medication management of patients with depression and anxiety can produce significant cost savings. Second, our model does not incorporate Quality of Life or Willingness to Pay Thresholds in our calculations. Future studies could develop cost-effectiveness models with varying time horizons and cost perspectives (payer, patient) to better approximate the costs and savings associated with pharmacogenetic testing. Models such as Markov or Discrete Event Simulation would allow for sensitivity analyses to assess the robustness of the model results and provide more reliable data.

\section{Conclusion}

A much-cited barrier for the implementation of personalized medicine is the lack of cost-effectiveness studies to assess the economic benefit of pharmacogenetic-guided medication management in a "real-world" environment. Our model presents significant cost savings of pharmacogenetic testing in a diverse set of clinical settings and health care providers. The clinical outcomes data from Bradley and the costsavings model presented here indicate that the clinical and economic benefits of pharmacogenetic testing are available to psychiatrists, primary care providers, gynecologists, and internists. Given the increased need for a variety of health care providers to prescribe and manage antidepressants, pharmacogenetic tests are a valuable tool that demonstrate improved patient outcomes in real-world settings and are strongly positioned to help reduce the economic burden of neuropsychiatric disorders.

\section{Acknowledgments}

The authors would like to thank Paul Greenberg and Mihran Yenikomshian for providing input and expertise that greatly assisted the research, and Marilyn Olson for her valuable feedback on the manuscript. This study was funded by AltheaDx.

\section{Author contributions}

$\mathrm{AM}$ and $\mathrm{AC}$ were involved in the study, design, data collection and analysis, and preparation of the manuscript. AL and JG were involved in the study design, interpretation of the results, and the review and revision of the manuscripts.
All authors contributed toward data analysis, drafting and revising the paper and agree to be accountable for all aspects of the work.

\section{Disclosure}

AM, AC, AL, and JG are employed by AltheaDx. The authors report no other conflicts of interest in this work.

\section{References}

1. Pratt LA, Brody DJ. Depression in the U.S. household population, 2009-2012. NCHS Data Brief. 2014;172:1-8.

2. Cuijpers P, Smit F. Excess mortality in depression: a meta-analysis of community studies. $J$ Affect Disorders. 2002;72(3):227-236.

3. Ruo B, Rumsfeld JS, Hlatky MA, Liu H, Browner WS, Whooley MA. Depressive symptoms and health-related quality of life: the heart and Soul Study. JAMA. 2003;290(2):215-221.

4. Khawaja IS, Westermeyer JJ, Gajwani P, Feinstein RE. Depression and coronary artery disease, the association, mechanisms and therapeutic implications. Psychiatry (Edgmont). 2009;6(1):38-51.

5. Zivin K, Ilgen MA, Pfeiffer PN, et al. Early mortality and years of potential life lost among veterans affairs patients with depression. Psychiatr Serv. 2012;63(8):823-826.

6. Chesney E, Goodwin GM, Fazel S. Risks of all-cause and suicide mortality in mental disorders: a meta-review. World Psychiatry. 2014 13(2):153-160.

7. Kessler RC, Frank RG. The impact of psychiatric disorders on work loss days. Psychol Med. 1997;27(4):861-873.

8. Broadhead WE, Blazer DG, George LK, Tse CK. Depression, disability days, and days lost from work in a prospective epidemiologic survey. JAMA. 1990;264(19):2524-2528.

9. Greenberg PE, Fournier AA, Sisitsky T, Pike CT, Kessler RC. The economic burden of adults with major depressive disorder in the United States (2005 and 2010). J Clin Psychiatry. 2015;76(2):155-162.

10. Simon Ge, VonKorff M, Barlow W. Health care costs of primary care patients with recognized depression. Arch Gen Psychiatry. 1995;52(10): 850-856.

11. U.S. Department of Health and Human Services, Centers for Disease Control and Prevention, National Center for Health Statistics. Health, United States, 2013: with special feature on prescription drugs. Available from: https://www.cdc.gov/nchs/data/hus/hus13.pdf. Accessed February 10, 2017.

12. Centerwatch.org [internet]. Boston, MA: Available from: https://www. centerwatch.com/drug-information/fda-approved-drugs/therapeuticarea/17/psychiatry-psychology. Accessed May 16, 2017.

13. Mrazek DA. Psychiatric pharmacogenomic testing in clinical practice. Dialogues Clin Neurosci. 2010;12(1):69-76.

14. Rush AJ, Trivedi MH, Wisniewski SR, et al. Acute and longer-term outcomes in depressed outpatients requiring one or several treatment steps: a STAR*D report. Am J Psychiatry. 2006;163(11):1905-1917.

15. Trivedi MH, Rush AJ, Wisniewski SR, et al; STAR*DStudy Team. Evaluation of outcomes with citalopram for depression using measurementbased care in STAR*D implications for clinical practice. Am JPsychiatry. 2006;163(1):28-40.

16. Huynh NN, McIntyre RS. What are the implications of the STAR*D Trial for primary care? A review and synthesis. Prim Care Companion J Clin Psychiatry. 2008;10(2):91-96.

17. Mental Health Care Health Professional Shortage Areas (HPSAs) The Henry J. Kaiser Family Foundation. Available from: http://kff.org/other/ state-indicator/mental-health-care-health-professional-shortage-areashpsas/?currentTimeframe=0\&sortModel=\%7B $\% 22$ colld $\% 22: \% 22$ Loca tion\%22,\%22sort\%22:\%22asc\%22\%7D. Accessed April 3, 2017.

18. 2012 Physician Specialty Data Book. Center for Workforce Studies. Washington, DC: Association of American Medical Colleges; 2012. 
19. Kessler RC, Berglund P, Demler O, Jin R, Merikangas KR, Walters EE. Lifetime prevalence and age-of-onset distributions of DSM-IV disorders in the National Comorbidity Survey Replication. Arch Gen Psychiatry. 2005;62(6):593-602.

20. Mark TL, Levit KR, Buck JA. Psychotropic drug prescriptions by medical specialty. Psychiatr Serv. 2009;60(9):1167.

21. Bradley P, Shiekh M, Mehra V, et al. Improved efficacy with targeted pharmacogenetic-guided treatment of patients with depression and anxiety: a randomized clinical trial demonstrating clinical utility. J Psychiatr Res. 2017;96:100-107.

22. Olson MC, Maciel A, Gariepy JF, etal. Clinical impact of pharmacogeneticguided treatment for patients exhibiting neuropsychiatric disorders: a randomized controlled trial. Prim Care Companion CNS Disord. 2017;19(2).

23. Hall-Flavin DK, Winner JG, Allen JD, et al. Utility of integrated pharmacogenomic testing to support the treatment of major depressive disorder in a psychiatric outpatient setting. Pharmacogenet Genomics. 2013;23(10):535-548.
24. Saldivar JS, Taylor D, Sugarman EA, et al. Initial assessment of the benefits of implementing pharmacogenetics into the medical management of patients in a long-term care facility. Pharmacogenomics Pers Med. 2016;9:1-6.

25. Zhou ZW, Chen XW, Sneed KB, et al. Clinical association between pharmacogenomics and adverse drug reactions. Drugs. 2015;75(6): 589-631.

26. Sugarman EA, Cullors A, Centeno J, Taylor D. Contribution of pharmacogenetic testing to modeled medication change recommendations in a long-term care population with polypharmacy. Drugs Aging. 2016; 33(12):929-936.

27. Winner J, Allen JD, Altar CA, Spahic-Mihajlovic A. Psychiatric pharmacogenomics predicts health resource utilization of outpatients with anxiety and depression. Transl Psychiatry. 2013;3:e242.

28. Chou WH, Yan FX, deLeon J, et al. Extension of a pilot study: impact from the cytochrome P4502D6 polymorphism on outcome and costs associated with severe mental illness. J Clin Psychopharmacol. 2000; 20(2):246-251.

\section{Publish your work in this journal}

Neuropsychiatric Disease and Treatment is an international, peerreviewed journal of clinical therapeutics and pharmacology focusing on concise rapid reporting of clinical or pre-clinical studies on a range of neuropsychiatric and neurological disorders. This journal is indexed on PubMed Central, the 'PsycINFO' database and CAS, and is the official journal of The International Neuropsychiatric Association (INA). The manuscript management system is completely online and includes a very quick and fair peer-review system, which is all easy to use. Visit http://www.dovepress.com/testimonials.php to read real quotes from published authors. 